\title{
Pharmacokinetics of oleracimine in rats by ultra-high-performance liquid chromatography
}

\author{
Gonglin $\mathbf{Q u}^{2}$, Liang Xu${ }^{1}$, Wenjie Zhang ${ }^{* 1}$, Xixiang Ying ${ }^{* 1}$ \\ ${ }^{1}$ School of Pharmacy, Liaoning University of Traditional Chinese Medicine, 116600 Dalian, China, ${ }^{2}$ National Institute for \\ Radiological Protection, China CDC, Beijing 100088, China
}

\begin{abstract}
The novel alkaloid, oleracimine, presented remarkable anti-inflammatory bioactivity, and therefore, its pharmacokinetics was investigated in rat plasma after intravenous and oral administration by using a rapid ultra-high-performance liquid chromatography (UHPLC) method with UV detection at $270 \mathrm{~nm}$. The analysis was performed on a shim-pack ODS column $(75 \mathrm{~mm} \times 2 \mathrm{~mm}, 1.6 \mu \mathrm{m}$ particle size, Shimadzu, Japan) column using isocratic elution with a mobile phase consisting of methanol-water $(62: 38, v / v)$ within $3 \mathrm{~min}$. The results indicated that oleracimine was rapidly distributed with $\mathrm{T}_{\max }$ for $11.7 \mathrm{~min}$ after oral administration, which presented the double-peak phenomenon in the pharmacokinetic profile with a higher oral absolute bioavailability of $55.1 \% \pm 7.83 \%$.
\end{abstract}

Keywords: Portulaca oleracea L./Oleracimine. Ultra-High-Performance Liquid Chromatography/Rat plasma. Pharmacokinetics.

\section{INTRODUCTION}

Portulaca oleracea L., a member of family Portulacaceae, is a warm climate, annual, green herb, with branched and succulent stems. As a widespread and abundant plant in the world, $P$. oleracea $\mathrm{L}$. is used as a folk medicine in many countries. P. oleracea L. is a well-known traditional Chinese medicine and used as a treatment for removing heat, counteracting toxicity, cooling blood, hemostasia, and antidysentery (Pharmacopoeia of PRC, 2015). Previous studies reported that $P$. oleracea L. presented many pharmacological properties, such as antioxidant (Chen et al., 2012), anti-inflammatory and analgesic (Chan et al., 2000), hypocholesterolemic (Movahedian, Ghannadi, Vashirnia, 2007), neuroprotective effects (Abdel Moneim, 2013), and antitumor (Zhao et al., 2013). Until now, the active compounds including terpenes (Xin et al., 2008), phenolic acids and coumarins (Cheng et al., 2012), flavonoids (Xu, Yu, Chen, 2006), and alkaloids (Jiao et al., 2015) were isolated from $P$. olerace $\mathrm{L}$., where the alkaloid has attracted many scholars' attention because of its stronger

*Correspondence: W. Zhang and X. Ying. School of Pharmacy, Liaoning University of Traditional Chinese Medicine, 116600 Dalian, China. Tel.: +86-0411-85890139 / Fax: +86-0411-85890128. E-mail: wenjiezhang509@hotmail.com / yingxixiang@163.com bioactivity among natural products. Recently, five novel alkaloids, namely, oleracone, oleracone A, oleracimine, oleracimine $\mathrm{A}$, and oleraciamide $\mathrm{C}$ have been isolated from $P$. oleracea L. in our laboratory, among which oleracimine presented remarkable anti-inflammatory effects on lipopolysaccharide-stimulated macrophages (Meng et al., 2016; Li et al., 2016; Xu et al., 2016). The results of enzyme-linked immunosorbent assay, Western blot, and real-time polymerase chain reaction showed that oleracimine remarkably inhibited nitric oxide production and could decrease the secretions of interleukin 6 , tumor necrosis factor $\boldsymbol{\alpha}$, nitric oxide, and prostaglandin E2 in cell culture supernatants, as well as the mRNA of cyclooxygenase- 2 and inducible nitric oxide synthase. Therefore, the pharmacokinetic profile of oleracimine is to be investigated by a rapid and specific ultra-highperformance liquid chromatography (UHPLC) in rat plasma after intravenous and oral administrations, and the absolute bioavailability of oleracimine was also discussed.

\section{MATERIAL AND METHODS}

\section{Material and reagents}

The whole herbs of $P$. oleracea $\mathrm{L}$. were collected in Shijiazhuang (Hebei, China) in June 2014. The plant was 
identified by Prof. Xixiang Ying. A voucher specimen (no. 20140312) was deposited at the School of Pharmacy, Liaoning University of Traditional Chinese Medicine. Oleracimine was isolated and purified in our laboratory (Li et al., 2017). For the internal standard (IS), bergapten was provided by Sichuan Weikeqi Biological Technology Co., Ltd (Chengdu, China) (Figure 1). Methanol and acetonitrile were all of HPLC grade provided by Damao Chemical Reagent Plant (Tianjin, China). All other reagents were of analytical grade and were purchased from Jinfeng Chemical Factory (Tianjin, China). Water was purified using Milli-Q ${ }^{\circledR}$ Biocel Ultrapure Water System (Millipore, Bedford, MA).

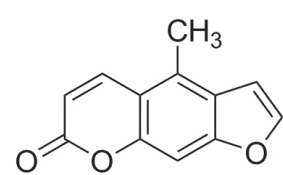

1

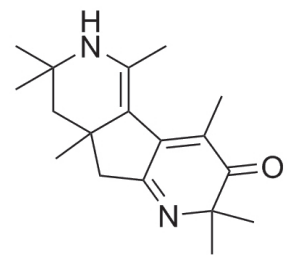

2
FIGURE 1 - Structures of internal standard (1) and oleracimine (2).

\section{Chromatographic system and conditions}

A Shimadzu UHPLC system (Japan) was equipped with a Solvent Delivery Pump (Shimadzu LC-30AD), a vacuum degasser (DGU-20A) a Shimadzu UV-VIS spectrophotometric detector (SPD-20A), and ChemStation software (Shimadzu). The analytical column was a shimpack ODS column $(75 \mathrm{~mm} \times 2 \mathrm{~mm}$; $1.6-\mu \mathrm{m}$ particle size; Shimadzu, Japan). The mobile phase for UHPLC analysis consisting of methanol-water $(62: 38, v / v)$ were passed under vacuum through a $0.22-\mu \mathrm{m}$ filter membrane and degassed by exposure to ultrasonic waves before use. UHPLC analysis with UV detection at $270 \mathrm{~nm}$ was performed at a flow rate of $0.3 \mathrm{~mL} / \mathrm{min}$, and the column temperature was $45^{\circ} \mathrm{C}$. The sample injection volume was $3 \mu \mathrm{L}$.

\section{Preparation of calibration standards and quality control (QC) samples}

The stock solutions and IS were prepared by dissolving in methanol to yield concentrations of $144 \mu \mathrm{g} /$ $\mathrm{mL}$ and $192 \mu \mathrm{g} / \mathrm{mL}$ respectively. The stock solution of IS was diluted with methanol to $3.84 \mu \mathrm{g} / \mathrm{mL}$ before use. Working standard solutions were freshly prepared by mixing and diluting the above mentioned stock solution with methanol at appropriate ratios to yield concentrations of $100.0,16.0,8.0,4.0,2.0,1.0,0.5$, and $0.25 \mu \mathrm{g} / \mathrm{mL}$ of oleracimine. The stock solution and working standard solutions were stored in a refrigerator. QC samples prepared at $0.75,7.5$, and $75.0 \mu \mathrm{g} / \mathrm{mL}$ of oleracimine in bulk and aliquots were stored frozen before use.

\section{Animals and blood sampling}

Male Wistar rats $(250 \pm 20 \mathrm{~g})$ were supplied by the Laboratory Animal Service Center of Dalian Medical University. They were kept in an environmentally controlled breeding room for 1 week before the experiments and were fed with regular chow with standard laboratory food and water ad libitum and fasted overnight before the experiment. All animal studies were performed according to the requirement of the National Act on the Use of Experimental Animal (China) that was approved by the Committee of Ethics of Animal Experimentation of Liaoning University of Traditional Chinese Medicine.

For the pharmacokinetics, the two groups of rat (five rats per group) were assigned to receive oleracimine solution via intravenous and oral administrations at a single dose of $2.5 \mathrm{mg} / \mathrm{kg}$, respectively. Serial blood samples $(0.3 \mathrm{~mL})$ were obtained via the orbitalis vena at intervals of $2,5,10,15,20,30,45,60,90,120,180$, and 240 min after administration and were collected in heparinized centrifuge tubes. The blood samples were immediately centrifuged at $3,500 \mathrm{~g}$ for $15 \mathrm{~min}$ at room temperature. The plasma sample was stored at $-20^{\circ} \mathrm{C}$ until analysis.

\section{Plasma sample preparation}

A $100-\mu \mathrm{L}$ plasma, $20-\mu \mathrm{L}$ IS $(3.84 \mu \mathrm{g} / \mathrm{mL})$, and 0.5 $\mathrm{mL}$ acetonitrile were added, followed by vortex mixing for $1 \mathrm{~min}$ and centrifuged at 3,500 $\mathrm{g}$ for $15 \mathrm{~min}$ to precipitate the protein. The supernatant was collected and evaporated to dryness at $40{ }^{\circ} \mathrm{C}$ under a gentle stream of nitrogen. The residue was reconstituted with $50 \mu \mathrm{L}$ mobile phase, and centrifuged at $16,350 \mathrm{~g}$ for $5 \mathrm{~min}$, and an aliquot $(3 \mu \mathrm{L})$ of the supernatant was injected into the UHPLC system.

\section{Method validation}

\section{Selectivity}

The selectivity was determined by comparing chromatograms of blank plasma obtained from the rats before dosing with the corresponding standard plasma spiked with the compound and IS and samples from rats after administration of the compound.

\section{Linearity, $L O D$, and $L O Q$}

The linearity was evaluated over the concentration 
range of 0.05 to $20 \mu \mathrm{g} / \mathrm{mL}$ at eight levels of the compound. The peak area ratios of the analyte with IS were plotted against the standard concentrations to establish calibration curves for oleracimine. The regression equation was obtained by using the $1 / \mathrm{X}^{2}$ weighted least squares linear regression method. The LOD and LOQ were determined by stepwise dilution of the QC sample at a low concentration level, using a signal-noise ratio of 3 and 10, respectively, which gives an acceptable accuracy (RE) within $\pm 20 \%$ and a precision (RSD) that did not exceed $20 \%$.

\section{Precision and accuracy}

The intraday and interday precisions and accuracy were, respectively, determined within 1 day and for three consecutive days by analyzing the QC samples at low, medium, and high concentrations. The intraday and interday precisions were defined as the relative standard deviation (RSD), and accuracy was determined by calculating the relative error (RE).

\section{Extraction recovery}

The RSD of each concentration was calculated to determine the precision of the method. The extraction recovery was determined in sets of five replicates of the QC samples by comparing the peak areas obtained for the QC samples at low, medium, and high concentrations, which were subjected to an extraction procedure with those obtained from blank plasma extracts that were spiked postextraction with the corresponding concentrations.

\section{Stability}

The stabilities were carried out by the QC samples at low, medium, and high concentrations. Short- and longterm stabilities were determined by analyzing the QC samples kept at ambient temperature $\left(25^{\circ} \mathrm{C}\right)$ for $4 \mathrm{~h}$ and stored at $-20^{\circ} \mathrm{C}$ for 1 month, respectively. Freeze-thaw stability was investigated after three freeze-thaw $\left(-20{ }^{\circ} \mathrm{C}\right.$ to room temperature) cycles. Then, the samples were processed and analyzed. The concentrations obtained were compared with the nominal values of the QC samples.

\section{Pharmacokinetic study}

Plasma data were subjected to analysis using both the statistical moment principle and the pharmacokinetic program, Version 1.0 (3p97) of the Chinese Society of Mathematical Pharmacology (Beijing, China). The maximum plasma concentration $\left(\mathrm{C}_{\max }\right)$ and the time to reach the maximum plasma concentration $\left(\mathrm{T}_{\max }\right)$ were obtained from 3 p97 pharmacokinetic program. The compartmental parameters were generated by the pharmacokinetic program, including $\alpha$ half-life $\left(\mathrm{t}_{1 / 2 \alpha}\right)$, $\beta$ half-life $\left(\mathrm{t}_{1 / 2 \beta}\right)$, clearance rate $(\mathrm{CL})$, and the area under the plasma concentration-time curve (AUC). The noncompartmental parameters and the area under the plasma concentration time curve $\left(\mathrm{AUC}_{0-t}\right)$ were calculated using the statistical moment method. The total area under the plasma concentration-time curve from time zero to time infinity $\left(\mathrm{AUC}_{0-\infty}\right)$ was calculated as the sum of $\mathrm{AUC}_{0-\mathrm{t}}$ and $\mathrm{C}_{\text {last }} / \mathrm{K}$, where $\mathrm{C}_{\text {last }}$ represents the last quantifiable concentration, and $\mathrm{K}$ represents the terminal phase rate constant. The terminal half-life $\left(\mathrm{t}_{1 / 2}\right)$ was calculated as $0.693 / \mathrm{K}$, and the $\mathrm{K}$ was estimated by natural logarithms of linear regression on the last five points. Clearance (CL) after dosing was calculated as dose $/ \mathrm{AUC}_{0-\infty}$. The mean retention time (MRT) was calculated though AUMC/AUC The absolute bioavailability $(\% \mathrm{~F})$ was calculated using the following formula:

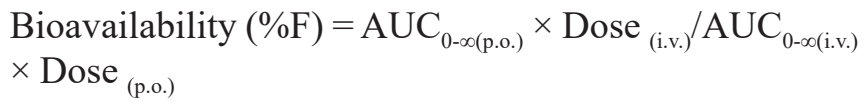

\section{RESULTS AND DISCUSSION}

\section{Method development and optimization}

During method development, the mobile phase composition varied using different combinations of methanol, acetonitrile, and water. Here, the mobile phase of methanol-water $(62: 38, v / v)$ was chosen to achieve good peak shape, satisfactory resolution, and relatively short analysis time. The UV absorption spectra of oleracimine have a maximum absorption at $270 \mathrm{~nm}$ and that of IS at $254 \mathrm{~nm}$. The interferences from endogenous substances in the plasma were observed when the wavelength was set at $254 \mathrm{~nm}$. Therefore, the detection wavelength was set at $270 \mathrm{~nm}$ because no interference appeared, and it was found to be suitable for the analysis of oleracimine and IS. To improve the accuracy of the method and reduce the error during injection process, the internal standard (IS) was chosen to analyze oleracimine. Baicalein, bergapten, kaempferol, and chrysophanol were, respectively, selected as candidates of IS, and only bergapten and chrysophanol had the close retention time with analyte, whereas chrysophanol presented a low recovery ratio which may be reduced by a high protein binding ratio of $85.7 \%$ (Feng et al., 2013), bergapten was therefore chosen as the internal standard. To simultaneously acquire high extraction recovery of analytes and IS, several solvents, including acetonitrile, methanol, and their different ratios, were applied to precipitate protein, only the satisfactory recoveries of analytes and IS $(81.9 \%$ and $86.3 \%)$ can be obtained simultaneously after acetonitrile was applied. 


\section{Method validation}

The representative chromatograms of blank plasma, plasma samples spiked with analyte and IS, and a plasma sample obtained from a rat after the intravenous and oral administration of oleracimine $(2.5 \mathrm{mg} / \mathrm{kg})$ are shown in Figure 2, indicating a good baseline resolution of IS and analyte at retention times of approximately $2.4 \mathrm{~min}$ and $2.7 \mathrm{~min}$, respectively. The total run time per sample was no more than $3 \mathrm{~min}$, and there were no interfering peaks of endogenous compounds in the chromatographic determinations. The regression equation of the calibration curves was typically: $y=0.936633 x+0.00443$, and the linear ranges for plasma were within 0.05 to $20 \mu \mathrm{g} / \mathrm{mL}$ with $r>0.9934$. The LOD $(\mathrm{S} / \mathrm{N}>3)$ and the LOQ $(\mathrm{S} / \mathrm{N}>$ 10) were, respectively, 0.017 and $0.05 \mu \mathrm{g} / \mathrm{mL}$ in plasma. The RSDs of precision and the REs of accuracy at low, medium, and high concentrations $(0.15,1.50,15.0 \mu \mathrm{g} / \mathrm{mL})$ were listed in Table I, and the results conform with the criteria for the analysis of biological sample according to the guidelines of USFDA (FDA, 2001). Mean extraction recovery at three concentration levels for oleracimine was $80.6 \pm 4.3 \%$ with an RSD of $8.5 \%$, suggesting negligible loss during extraction. The short-, long-term, and freezethaw stability of analytes in plasma ranged from $80.1 \%$ to
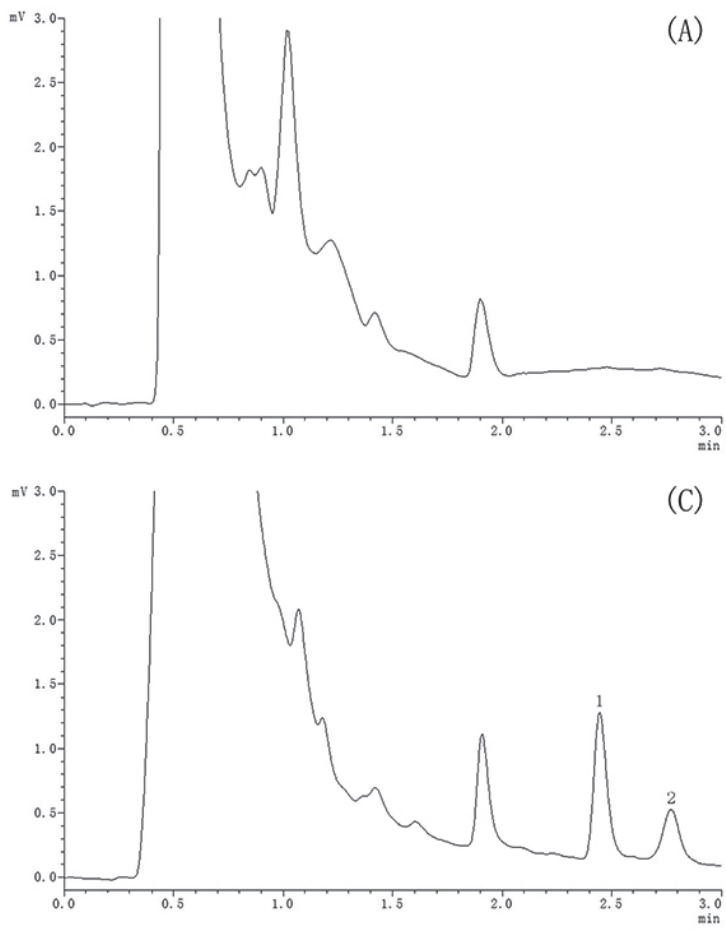

$83.4 \%$, indicating that no remarkable degradation occurred during chromatography, extraction, and storage processes for the plasma samples.

\section{Pharmacokinetics}

The pharmacokinetic parameters were estimated by compartmental and noncompartmental analyses, and all the pharmacokinetic parameters are given in Table II. According to the AIC and $\mathrm{R}^{2}$, a two-compartment open model (weight $=1$ ) gave the best fit to the plasma concentration-time curves obtained in rats. The mean plasma concentration-time curves of oleracimine in rat after oral and intravenous administrations at a dose of $2.5 \mathrm{mg} / \mathrm{kg}$ are shown in Figure 3, which showed that the concentration-time curves of oleracimine presented the double-peak phenomenon in the pharmacokinetic profile, and two mechanisms should be responsible for the phenomenon: (1) enterohepatic recycling, the cycle in which bile salts and drugs excreted by the liver are absorbed by the intestinal mucosa and returned to the liver via the portal circulation (Zhu et al., 2010). 2) For absorption difference, the lipophilic drug was rapidly distributed into the organs at first and was released to the plasma again after the metabolism of the drug has reached
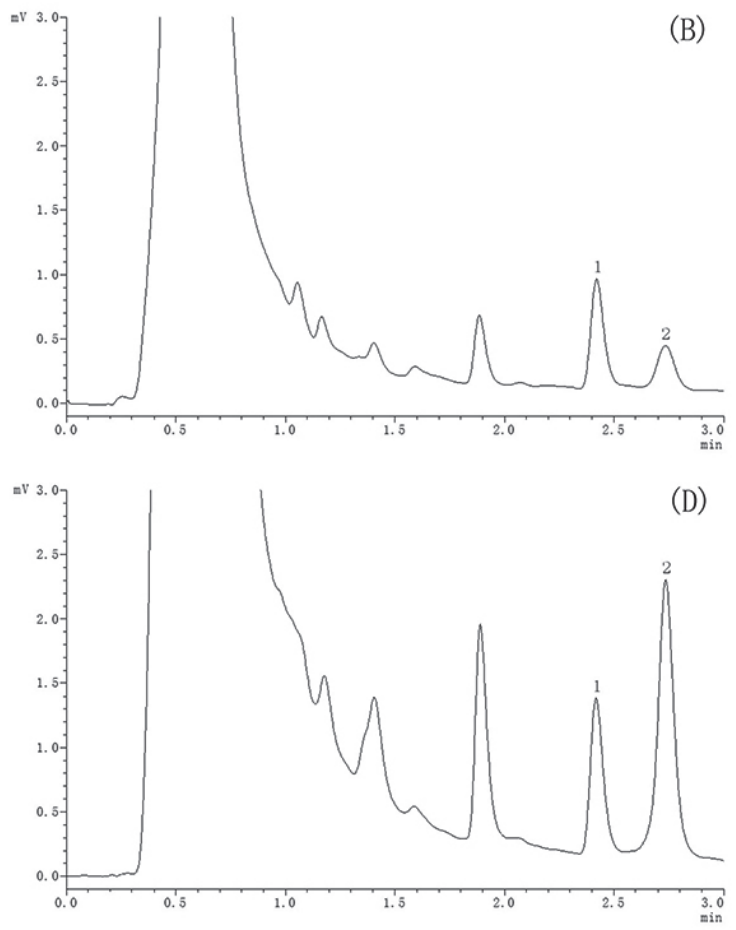

FIGURE 2 - (A) - Representative chromatogram of blank plasma. (B) - Representative chromatogram of plasma sample 20 min after the intravenous administration. (C) - Representative chromatogram of oral administration of oleracimine at a dose of $2.5 \mathrm{mg} / \mathrm{kg}$. (D) - Representative chromatogram of plasma spiked with oleracimine and IS. Peak 1: Internal standard; Peak 2: oleracimine. 
TABLE I - Precisions and accuracies of oleracimine in rat plasma (intra-day: $n=5$; inter-day: $n=3$ days with 5 replicates per day)

\begin{tabular}{|c|c|c|c|c|c|c|}
\hline \multirow[b]{2}{*}{$\begin{array}{l}\text { Added conc. } \\
(\mu \mathrm{g} / \mathrm{mL})\end{array}$} & \multicolumn{3}{|c|}{ Intra-day } & \multicolumn{3}{|c|}{ Inter-day } \\
\hline & $\begin{array}{c}\text { Conc. } \\
(\mu \mathrm{g} / \mathrm{mL}) \\
(\mathrm{mean} \pm \mathrm{SD})\end{array}$ & $\begin{array}{c}\text { R.S.D. } \\
(\%)\end{array}$ & $\begin{array}{l}\text { R.E. } \\
\text { (\%) }\end{array}$ & $\begin{array}{c}\text { Conc. } \\
(\mu \mathrm{g} / \mathrm{mL}) \\
(\operatorname{mean} \pm \mathrm{SD})\end{array}$ & $\begin{array}{c}\text { R.S.D. } \\
(\%)\end{array}$ & R.E. (\%) \\
\hline 0.15 & $0.154 \pm 0.013$ & 8.70 & 2.4 & $0.146 \pm 0.024$ & 9.90 & -2.7 \\
\hline 1.5 & $1.49 \pm 0.061$ & 4.07 & -0.60 & $1.37 \pm 0.093$ & 7.89 & -8.7 \\
\hline 15 & $14.9 \pm 0.32$ & 2.15 & -0.80 & $14.5 \pm 0.57$ & 4.56 & -3.3 \\
\hline
\end{tabular}

a certain degree, resulting in the appearance of double peaks (Ma, Sun, 1993). Also, oleracimine was rapidly distributed and eliminated in rat plasma with a $\mathrm{T}_{\max }$ of $11.7 \mathrm{~min}$ and $\mathrm{a} \mathrm{T}_{1 / 2}$ of $75.2 \mathrm{~min}$ after oral administration. $\mathrm{AUC}_{0 \rightarrow \infty}$ was $76.2 \pm 6.2 \mu \mathrm{g} \cdot \mathrm{min} / \mathrm{mL}$ intravenous route is larger than $38.9 \pm 3.1 \mu \mathrm{g} \cdot \mathrm{min} / \mathrm{mL}$ oral route, suggesting that the intravenous route had higher absorption than oral route. Besides the apparent volumes of distribution after both administrations (intravenous: $\mathrm{V}_{\mathrm{d}}=1.09 \pm 0.20$ $\mathrm{L} / \mathrm{kg}$, oral: $\mathrm{V}_{\mathrm{d}}=2.08 \pm 0.30 \mathrm{~L} / \mathrm{kg}$ ) were very small, showing that oleracimine distributed mainly in plasma. The CL of the intravenous route $(9.8 \pm 0.8 \mathrm{~mL} / \mathrm{min} \cdot \mathrm{kg})$ was smaller than that of the oral route $(19.3 \pm 1.4 \mathrm{~mL} /$ $\min \cdot \mathrm{kg}$ ), indicating that oleracimine after intravenous administration eliminated slower in the blood than the oral administration, and oral administration would

TABLE II - Pharmacokinetic parameters of oleracimine in rat (mean \pm S.D., $n=5$ ) following oral and intravenous routes of administration at dose of $2.5 \mathrm{mg} / \mathrm{kg}$

\begin{tabular}{lcc}
\hline Parameter & $\begin{array}{c}\text { Intravenous route } \\
(\boldsymbol{n}=\mathbf{5})\end{array}$ & $\begin{array}{c}\text { Oral route } \\
(\boldsymbol{n}=\mathbf{5})\end{array}$ \\
\hline $\mathrm{C}^{\mathrm{a}}{ }_{\max }(\mu \mathrm{g} / \mathrm{mL})$ & - & $0.346 \pm 0.013$ \\
$\mathrm{~T}^{\mathrm{a}}{ }_{\max }(\mathrm{min})$ & - & $11.7 \pm 0.86$ \\
$\mathrm{t}^{\mathrm{a}}{ }_{1 / 2 \alpha}(\mathrm{min})$ & $2.62 \pm 0.15$ & $4.68 \pm 0.21$ \\
$\mathrm{t}^{\mathrm{a}}{ }_{1 / 2 \beta}(\mathrm{min})$ & $65.4 \pm 4.7$ & $106 \pm 5.7$ \\
$\mathrm{t}^{\mathrm{b}}{ }_{1 / 2}(\mathrm{~min})$ & $77.4 \pm 6.1$ & $75.2 \pm 4.6$ \\
${ }^{\mathrm{b}} \mathrm{V}_{\mathrm{d}}(\mathrm{L} / \mathrm{kg})$ & $1.09 \pm 0.20$ & $2.08 \pm 0.30$ \\
$\mathrm{k}(1 / \mathrm{min})$ & $0.009 \pm 0.001$ & $0.009 \pm 0.0006$ \\
$\mathrm{AUC}{ }^{\mathrm{a}}(\mathrm{min} \cdot \mu \mathrm{g} / \mathrm{mL})$ & $75.6 \pm 5.2$ & $45.1 \pm 3.8$ \\
$\mathrm{AUC} \mathrm{C}^{\mathrm{b}}(\mathrm{min} \cdot \mu \mathrm{g} / \mathrm{mL})$ & $76.2 \pm 6.2$ & $38.9 \pm 3.1$ \\
$\mathrm{CL}^{\mathrm{b}}(\mathrm{mL} / \mathrm{min} \cdot \mathrm{kg})$ & $9.8 \pm 0.8$ & $19.3 \pm 1.4$ \\
$\mathrm{MRT}(\mathrm{min})$ & $111 \pm 12$ & $108 \pm 8.2$ \\
$\mathrm{~F}^{\mathrm{b}}(\%)$ & - & $55.1 \pm 7.83$ \\
\hline
\end{tabular}

a,b The compartmental and non-compartmental approach, respectively.

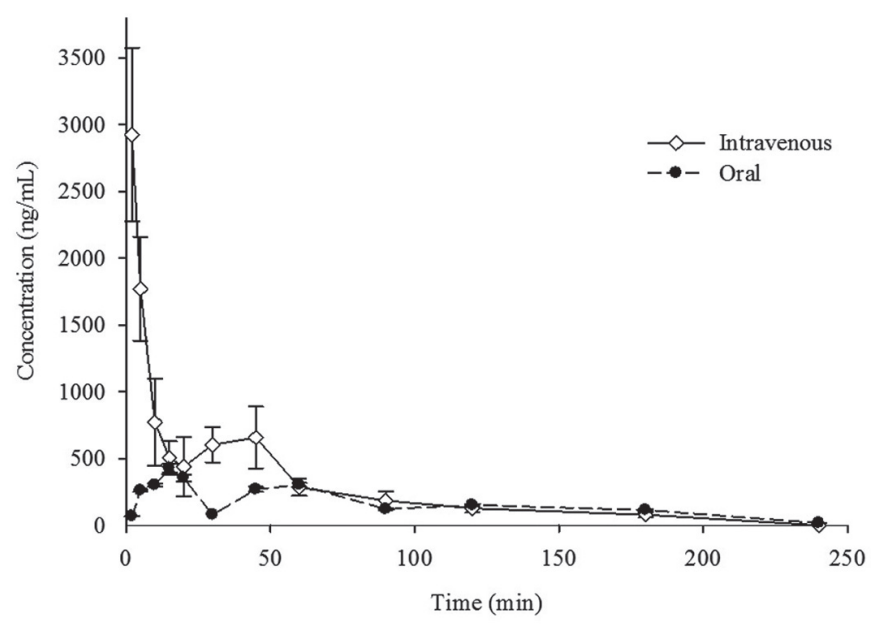

FIGURE 3 - Mean plasma concentration-time curves of oleracimine in rats (mean \pm S.D., $n=5$ ) after the intravenous and oral administrations at a dose of $2.5 \mathrm{mg} \cdot \mathrm{kg}^{-1}$. These sampling points, which could not be detected, that is, plasma concentrations under the LOQ, were not included in the mean plasma concentration-time curves.

be better for the metabolic process of oleracimine in the body. In addition, oleracimine has a higher oral bioavailability of $55.1 \pm 7.83$, suggesting that oleracimine is of significance for the pharmaceutical industry because of its anti-inflammatory bioactivity and higher bioavailability.

\section{CONCLUSIONS}

The pharmacokinetics of the novel alkaloid, oleracimine was investigated after intravenous and oral administrations using a rapid and specific UHPLC method for the first time. Oleracimine presented the double-peak phenomenon in the pharmacokinetic profile and a higher oral absolute bioavailability. Taken together, our study will provide reliable scientific data for its application as the leading compound to treat inflammatory diseases in clinical therapeutic approach in the future. 


\section{ACKNOWLEDGMENTS}

This study was supported by the Project of National Natural Science Foundation of China (Grant 81573546) and the Natural Science Foundation of Liaoning Province (Grant 2015020699), China.

\section{REFERENCES}

Abdel Moneim, AE. The neuroprotective effects of purslane (Portulaca oleracea) on rotenone induced biochemical changes and apoptosis in brain of rat. CNS Neurol Disord Dr. 2013;12(6):830-841.

Chan K, Islam MW, Kamil M, Radhakrishnan R, Zakaria MN, Habibullah M, et al. The analgesic and anti-inflammatory effects of Portulaca oleracea L subsp. sativa (Haw.) Celak. J Ethnopharmacol. 2000;73(3): 445-451.

Chen B, Zhou H, Zhao W, Zhou W, Yuan Q, Yang G. Effects of aqueous extract of Portulaca oleracea $\mathrm{L}$. on oxidative stress and liver, spleen leptin, PAR and FAS mRNA expression in high-fat diet induced mice. Mol Biol Rep. 2012;39(8):7981-7988.

Cheng ZZ, Xie M, Zhang WJ, Cheng L, Du Y, Wang YJ, et al. HPLC method for the simultaneous determination of four compounds in rat plasma after intravenous administration of Portulaca oleracea L. extract. Braz J Pharm Sci. 2012;48(1):16370.

Feng SX, Wu J, LI JS, Qu LB, Xu HP, Yang R, et al. Determination of the binding rate of rat plasma protein with five anthraquinones in rhubarb. Chin J Mod Appl Pharm. 2013;30:1-5.

Jiao ZZ, Yue S, Sun HX, Jin TY, Wang HN, Zhu RX, et al. Indoline amide glucosides from Portulaca oleracea: Isolation, structure, and DPPH radical scavenging activity. J Nat Prod. 2015;78(11):2588-2597.

Movahedian A, Ghannadi A, Vashirnia M. Hypocholesterolemic effects of purslane extract on serum lipids in rabbits fed with high cholesterol levels. Int J Pharmacol. 2007;3(3):285-289.

Li CY, Meng YH, Ying ZM, Xu N, Hao D, Gao MZ, et al. Three novel alkaloids from Portulaca oleracea L. and their antiinflammatory effects. J Agric Food Chem. 2016;64(29):58375844.
Li CY, Meng YH, Ying ZM, Xu N, Hao D, Gao MZ, et al. Correction to Three novel alkaloids from Portulaca oleracea L. and their antiinflammatory effects. J Agric Food Chem. 2017;65(4): 993-994.

Ma YM, Sun RY. Second peak of plasma diazepam concentration and enterogastric circulation. Acta Pharmacol Sin. 1993;14(3):218-221.

Meng YH, Ying ZM, Xiang Z, Hao D, Zhang WJ, Zheng Y, et al. The anti-inflammation and pharmacokinetics of a novel alkaloid from Portulaca oleracea L. J Pharm Pharmacol. 2016;68(3):397-405.

Pharmacopoeia of the People's Republic of China, in Part 1. Beijing: China Medical Science and Technology Press; 2015. p.49-50.

Xin HL, Xu YF, Hou YH, Zhang YN, Yue XQ, Lu JC, et al. Two novel triterpenoids from Portulaca oleracea L. Helv Chim Acta, 2008;91:2075-80.

Xu L, Ying ZM, Wei WJ, Hao D, Wang HB, Zhang WJ, et al. A novel alkaloid from Portulaca oleracea L. Nat Prod Res. 2016;31(8):902-908.

Xu XQ, Yu LS, Chen GN. Determination of flavonoids in Portulaca oleracea L. by capillary electrophoresis with electrochemical detection. J Pharmaceut Biomed. 2006;41(2):493-499.

Zhao R, Gao X, Cai YP, Shao XY, Jia GY, Huang YL, et al. Antitumor activity of Portulaca oleracea L. polysaccharides against cervical carcinoma in vitro and in vivo. Carbohydr Polym. 2013;96(2):376-383.

Zhu Z, Zhao L, Liu X, Chen J, Zhang H, Zhang G, et al. Comparative pharmacokinetics of baicalin and wogonoside by liquid chromatography-mass spectrometry after oral administration of Xiaochaihu Tang and Radix scutellariae extract to rats. J Chromatogr B. 2010;878(24):2184-2190.

Received for publication on $26^{\text {th }}$ April 2017 Accepted for publication on $28^{\text {th }}$ March 2018 